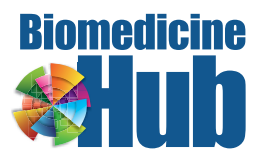

\title{
The Role of Multigene Assays in
} Addressing Treatment Heterogeneity for Breast Cancer

\author{
Matti Aapro \\ Clinique de Genolier, Breast Center, Genolier, Switzerland
}

\section{Keywords}

Breast cancer · Treatment heterogeneity $\cdot$ Chemotherapy · Multigene assay

\begin{abstract}
Breast cancer has a complex biology with heterogeneous treatment approaches that have changed significantly over the last two decades. The MAGIC survey provides valuable insight into worldwide treatment recommendations for early breast cancer patients and the clinical and pathologic criteria used for these decisions. The overall findings indicate that there is substantial heterogeneity in how patients are treated and a substantial uncertainty in treatment recommendations for a large proportion of patients, highlighting an unmet need for broadly available markers, such as multigene assays, that can help to make more informed treatment decisions by predicting a patient's likelihood of benefit from adjuvant chemotherapy.

(C) 2017 The Author(s)

Published by S. Karger AG, Basel
\end{abstract}

Breast cancer is the malignancy with the highest incidence among women in the Western world [1]. It has a complex biology with heterogeneous treatment approaches that have changed significantly over the last two decades.

Hormone therapy has long been a weapon in the physician's armamentarium for tackling malignant breast tumours. Chemotherapy and targeted therapy - for instance, with trastuzumab - also offer the prospect of improving survival rates among some subgroups of patients. The benefit of adding chemotherapy to hormonal therapy was limited to a modest number of patients [2], but because it was not possible to identify these patients specifically, physicians had to adopt an approach of treating many - even among node-negative, relatively low-risk groups - in the hope of improving the prospects for these few.

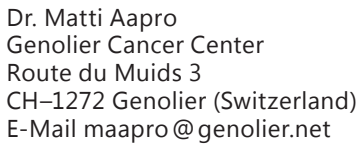


The physical and psychological side effects of this therapy take a substantial toll on patients - it is a highly undesirable ordeal for those who do not need it. So, the challenge for the medical community has been to pinpoint that population which truly does carry a risk of recurrence and where chemotherapy adds to hormonal therapy because of the biological tumour characteristics.

Traditional measures for categorising patients - parameters such as age, grade of tumour, nodal status, and size of tumour - are only prognostic at the wider population level and are not absolute predictors of a chemotherapy benefit. And attitudes towards chemotherapy tend to vary between clinicians - some lean towards being "chemocentric," while others could be characterised as "chemo-avoiders."

The Multidisciplinary Application of Genomics in Clinical Practice (MAGIC) survey [3] brings out these discrepancies clearly. Conducted between August 2013 and January 2014, the survey results were based on the responses of 911 practising clinicians and pathologists from 52 countries, mostly in Europe. The survey asked respondents to indicate 1 of 3 treatment recommendations for patients with early-stage hormone-positive breast cancer chemotherapy in addition to endocrine treatment, endocrine treatment alone, or a request for more information - based on 7 patient characteristics. Each physician was asked to indicate treatment recommendations for 24 breast cancer patient profiles varying in age, tumour size, tumour grade, oestrogen receptor, progesterone receptor, and Ki-67 expression, and lymph node status. The survey found that for $52 \%$ of the patient profiles, there was a wide range of recommendations, with at least every fourth physician recommending a different treatment pathway.

There is a manifest need for better evidence to inform treatment decisions and greater consistency in clinical practice. This is where multigene assays can play a key role. By analysing genes within a tumour sample, genomic tests can provide additional information about the likelihood of a cancer recurring following surgery to remove the tumour. A number of these assays have been developed, but, at the time of the MAGIC survey, the most commonly used test in 12 out of 14 countries was the Oncotype DX Breast Recurrence Score ${ }^{\circledR}$, which measures the expression of 21 genes in a tumour sample removed during the original surgery. A series of studies have shown that multigene assays can be accurate indicators of recurrence risk and have the potential to influence a significant proportion of clinicians' treatment decisions. Yet, international uptake remains patchy.

We should bear in mind that the data in the MAGIC survey are 3 years old. Nevertheless, they show that at the time of asking, 55\% of the physicians surveyed had adopted multigene assays in their practice. That adoption varied strongly between countries: in Greece, 91\% of the physicians surveyed used them, and in Germany, Argentina, and the Netherlands, more than 8 out of 10 doctors had adopted them to some extent. Yet, in Sweden, the tests were barely used at all, in Italy the figure was less than a quarter, and in Russia and the UK only around a third of the doctors used them. The study found that in all countries except Germany (50\%), the majority of the physicians who did not use multigene assays would like to use these tests. Lack of reimbursement, the price, and lack of availability were cited as the main barriers to usage.

The role of multigene assays in narrowing the field for chemotherapy by identifying the subset of patients at low risk who can be spared such treatment is becoming more established. However, the question remains as to whether we have the opportunity to be more ambitious: rather than merely ruling out a low-risk group of patients from chemotherapy, can we aim to predict who these patients are, by zeroing in specifically on the relatively few people who are most likely to derive a benefit?

If this is the goal, it necessitates using a marker that is specifically predictive of a response to chemotherapy treatment. Only one multigene assay is presently validated to do this: the 
Oncotype DX Breast Recurrence Score ${ }^{\circledR}$, as was also voted at the St. Gallen International Breast Cancer Conference [4] in Vienna in 2017. This method yields a score from 0 to 100, indicating the likely benefit from chemotherapy and the likelihood of cancer recurring within 10 years of diagnosis.

For any novel technological breakthrough, there are short-term cost barriers to adoption. Yet, targeting the right patient with the right treatment ultimately offers major benefits in both clinical and economic terms. Multigene assays are an example of personalised medicine in action, and they can play a key role in creating a recognised standard of clinical excellence in clinicians' recommendations for breast cancer treatment.

Despite the promise they offer, multigene assays are not yet fully embedded in clinical practice - especially in Europe, where reimbursement and value assessment remain the key barriers to equal access for patients. New pricing and reimbursement models which reward innovation and value are urgently needed to accelerate the adoption of these tests and to ensure that patients and physicians can make informed treatment decisions leading to improved clinical outcomes.

\section{Disclosure Statement}

The author is a consultant to Genomic Health Inc.

\section{Funding Sources}

The MAGIC survey was supported by an unrestricted grant from Genomic Health Inc., Redwood City, CA, USA. The sponsor was involved in the study design and writing of the study report.

\section{References}

1 International Agency for Research on Cancer, EUCAN: Breast cancer: estimated incidence, mortality and prevalence, 2012. 2012. http://eco.iarc.fr/EUCAN/CancerOne.aspx?Cancer=46\&Gender=2.

-2 Fisher B, Dignam J, Wolmark N, DeCillis A, Emir B, Wickerham DL, Bryant J, Dimitrov NV, Abramson N, Atkins JN, Shibata H, Deschenes L, Margolese RG: Tamoxifen and chemotherapy for lymph node-negative, estrogen receptor-positive breast cancer. J Natl Cancer Inst 1997;89:1673-1682.

-3 Aapro M, De Laurentiis M, Rea D, Bargallo Rocha JE, Elizalde R, Landherr L, Linderholm B, Mamounas E, Markopoulos C, Neven P, Petrovsky A, Rouzier R, Smit V, Svedman C, Schneider D, Thomssen C, Martin M: The MAGIC survey in hormone receptor positive (HR+), HER2-negative (HER2-) breast cancer: when might multigene assays be of value? Breast 2017;33:191-199.

-4 Curigliano G, Burstein HJ, Winer EP, Gnant M, Dubsky P, Loibl S, et al: De-escalating and escalating treatments for early stage breast cancer: the St. Gallen International Expert Consensus Conference on the Primary Therapy of Early Breast Cancer 2017. Ann Oncol 2017;28:1700-1712. 